\section{ROLE OF COMMUNITY PHARMACIST IN PHARMACOVIGILANCE}

\author{
Nayab Farooq ${ }^{\boxplus}$, Fatima Amin'
}

THIS ARTICLE MAY BE CITED AS: Farooq N, Amin F. Role of community pharmacist in pharmacovigilance. Khyber Med Univ J 2019; I I(3): I82-3 DOI: 10.35845/kmuj.2019.18589

W orld Health Organization ( $\mathrm{W} \mathrm{HO}$ ) def i n e s pharmacovigilance as the detection, assessment, understanding and prevention of adverse drug reactions or any other possible drug related problems.' Pharmacovigilance is important for the safe and efficient usage of drugs. Pharmacovigilance is just not an indicator it's a discipline for detecting and reporting ADRs. ${ }^{2,3}$

In 1968 after thalidomide tragedy (phocomelia in babies, whose mothers have taken thalidomide during pregnancy) WHO established adverse drug reactions (ADRs) reporting system. WHO defines ADR as any response to a drug which is harmful and accidental and occurs at doses that are normally used in human for prophylaxis, diagnosis, treatment or the mitigation of disease.'

The Uppsala Monitoring Centre (UMC) is the WHO cooperating center for global pharmacovigilance. The aim of UMC is to support and publicize patient protection through efficacious and worldwide pharmacovigilance practice. It provides autonomous and high excellent medical reference sources universally. It encourages indigenous pharmacovigilance initiatives by giving approach to passable resources and providing appropriate assistance.'

Pre marketing clinical trials do not give the sufficient knowledge about adverse drug reactions because the minimum number of population is included in these clinical trials and exposure time of targeted drug is also short so clinical trials are unable to detect those ADRs who are race specific or occur in I/I000 or $1 / 10,000$ ratio. Post marketing surveillance and pharmacovigilance are the essential steps to ensure the safety and efficacy of drug. ${ }^{4,5}$

\section{Pakistan Framework}

The government of Pakistan in collaboration with drug regulatory authority of Pakistan (DRAP) has started the online pharmacovigilance (PVRSD) system in 2017. This program collects information from medicals professionals, pharmaceutical companies and patients on ADRs.

\section{Role of Pharmacist}

The professional responsibility of community pharmacist is far more than just preparing and dispensing medicines. Pharmacist can play a patient centric role by ensuring the safety of medicines through pharmacovigilance. Community pharmacists can ensure the safe and efficacious use of medicines by identifying and commentating ADRs. ${ }^{4}$

Community pharmacists can play there part in safe usage of medication and prevention of ADRs.' The data composed by community pharmacists on pharmacovigilance is worthwhile. ${ }^{7}$

The role of pharmacist in pharmacovigilance differs from state to state but the professional concern remains similar irrespective of territories. ${ }^{6}$ Community pharmacists can report ADRs based on their clinical experience and interacting and counseling with the patients at community pharmacy. The pharmacovigilance program of government can only be successful if the community pharmacists play their role actively in reporting ADRs. The most important step for the pharmacovigilance is to inform the all healthcare practitioners.'

Community pharmacists can act as a bridge between physicians and patients to ensure drug safety and efficacy. Community pharmacists can prevent
I M. Phil Scholar, Institute of Pharmacy, Lahore College for Women University, Lahore, Pakistan

Email»: nayabfarooq49@gmail.com

\section{Date Submitted:}

August 31, 2018

Date Revised: September 20, 2019

Date Accepted: September 23, 2019

ADRs by counseling with patients and providing them communication materials newsletters and other drug information resources utilized by other healthcare professionals for drug safety information. $^{8}$

Community pharmacists can educate and counsel patients in order to reduce medication errors and to improve the patient's quality of life. ${ }^{8}$

In our community pharmacy setting most of the pharmacists do no report ADRs because they are not acquainted with the ADR reporting process.'

Apart from reporting ADRs community pharmacists can play their role by keeping the record of identified ADRs at their workplace. Community pharmacists can also contribute in drug safety by updating their knowledge regarding new drug inventions and aspects of drugs. For pharmacovigilance pharmacist should have a sound regarding the usage of over the counter medications. $^{6}$

Pharmacovigilance knowledge can strengthen the role of community pharmacist in a productive way. Educational trainings and workshops can improve the understanding of community pharmacists regarding ADR identification and reporting. This can assist the community pharmacists to perform a better part in pharmacovigilance and safe usage of medications.

\section{REFERENCES}

I. World Health Organization (WHO). The importance of Pharmacovigilance - Safety Monitoring of Medicinal Products. WHO, Geneva. 2002

2. Yamamoto M. Patient Drug Information Leaflets for Risk/Benefit C $\circ \mathrm{m} \mathrm{m} \mathrm{u} \mathrm{n} \mathrm{i} \mathrm{c} \mathrm{a} \mathrm{t} \mathrm{i} \circ \mathrm{n}$. J Pharmacovigil20I4;3(I): 1000el 32. 
DOI: $10.4 \mid 72 / 2329-6887.1000 e$ 132

3. The Upssala monitoring center. Building a global patient safety culture. [Cited on: August 20, 2018]. Available from URL: https://www.who-umc.org/aboutus/who-we-are/our-vision-andmission/

4. Granas AG, Buajordet M, StenbergNilson H, Harg P, Horn AM. Pharmacists attitudes towards the reporting of suspected adverse drug reactions in Norway. Pharmacoepidemiol Drug Saf 2007;16(4):429-34. DOI:

\subsection{2/pds. 1298}

5. Zolezzi M, Parsotam N. Adverse drug reaction reporting in $\mathrm{New}$ Zealand: implications for pharmacists. Ther Clin Risk Manag 2005: I (3): I8I-8.

6. Drug regulatory of Pakistan. Guidelines undertaken for performance of Pharmacovigilance activities. [Cited on: August 20, 2018]. Available from URL: http://dra.gov.pk/Home/Download sAllDocs

7. Rama P, Prudence RA, Archana G. Pharmacovigilance: Perspectives and future challenges in Indian scenario. Asian J Pharm Clin Res 20I I;4(4): I-4.

8. Rajanandh MG, Praveen Kumar V, Yuvasakthi S. Roles of Pharmacist in Pharmacovigilance: A Need of the Hour. J Pharmacovigil 2016;4(6): I00022I. DOI: 10.4I72/23296887.1000221

9. Schumacher C. Pharmacist Involvement in a Patient-Centered Medical Home. Pharmacy Practice Model Initiative (PPMI) Case Study. [Cited on: August 20, 2018]. Available from URL: http://www.ashpmedia.org/pai/doc s/casestudy-Midwestern.pdf
CONFLICT OF INTEREST

Authors declared no conflict of interest GRANT SUPPORT AND FINANCIAL DISCLOSURE NIL

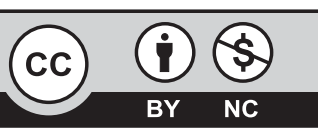

This is an Open Access article distributed under the terms of the Creative Commons Attribution-Non Commercial 2.0 Generic License.

KMUJ web address: www.kmuj.kmu.edu.pk

Email address: kmuj@kmu.edu.pk 